\title{
Jaw Clicking or Popping
}

National Cancer Institute

\section{Source}

National Cancer Institute. Jaw Clicking or Popping. NCI Thesaurus. Code C94559.

Noise in the temporomandibular joint, with or without pain, when fully opening the jaw. 